\title{
Face Mask Detection System using Deep Learning
}

\author{
Pinki $^{1}$ | Prof. Sachin Garg ${ }^{2}$ \\ ${ }^{1}$ B.Tech Scholar, Department of IT, Maharaja Agrasen Institute of Technology, Delhi, India \\ ${ }^{2}$ Assistant Professor, Department of IT, Maharaja Agrasen Institute of Technology, Delhi, India
}

\section{To Cite this Article}

Pinki and Prof. Sachin Garg, "Face Mask Detection System using Deep Learning", International Journal for Modern Trends in Science and Technology, 6(12): 161-164, 2020.

\section{Article Info}

Received on 08-November-2020, Revised on 28-November-2020, Accepted on 02-December-2020, Published on 05-December-2020.

\section{ABSTRACT}

In the present scenario due to Covid-19, there is no efficient face mask detection applications which are now in high demand for transportation means, densely populated areas, residential districts, large-scale manufacturers and other enterprises to ensure safety. This system can therefore be used in real-time applications which require face-mask detection for safety purposes due to the outbreak of Covid-19. This project can be integrated with embedded systems for application in airports, railway stations, offices, schools, and public places to ensure that public safety guidelines are followed. To identify the person on image/video stream wearing face mask or not. If the person doesn't wear a mask, the notification will be sent to the respected admin with the help of Python and deep learning algorithm by using the Convolutional Neural Network, Keras Framework and OpenCV.

KEYWORDS: Computer Vision, Object Detection, Object Tracking, COVID-19, Face Masks, Safety Improvement.

\section{INTRODUCTION}

In this paper, I have developed a deep learning model for face mask detection using Python, Keras, and OpenCV. We developed the face mask detector model for detecting whether person is wearing a mask or not. We have trained the model using Keras with network architecture. Training the model is the first part of this project and testing using webcam using OpenCV is the second part.

In order to use face mask detection system, we need to begin with an image of a person who does not wear a facial mask:

1. We apply face detection from there to calculate the location of the bounding box in the image.

2. Once we know where in the image the face is, we can extract the face Region of Interest (ROI), and from there, we apply facial landmarks, allowing us to localize mouth, face, and eyes.

3. In order to apply masks, we need an image of a mask (with a transparent and high definition image). Add the mask to the detected face and then resize and rotate, placing it on the face.

4. Repeat this process for all input image.

\section{RELATED WORK}

Under the current Covid-19 lockdown time such system is definitely important to prevent the spread in many use cases. Following are few use cases which will benefit out of this system.

- Offices - Manufacturers, retail, other SMEs and corporate giants

- Hospitals/healthcare organizations

- Airports and railway stations 
- $\quad$ Sports venues

- Entertainment and hospitality industry

- Densely populated areas

Analyzing the current scenario, government and private organizations want to make sure that everyone working or visiting a public or private place is wearing masks throughout the day. The face mask detection platform can quickly identify the person with a mask, using cameras and analytics.

\section{The system has been worked with the following} classifiers:

MobilenetV2: MobileNetV2 is a state of the art for mobile visual recognition including classification, object detection and semantic segmentation. This classifier uses Depth wise Separable Convolution which is introduced to dramatically reduce the complexity cost and model size of the network, and hence is suitable to Mobile devices, or devices that have low computational power. In MobileNetV2, another best module that is introduced is inverted residual structure. Non-linearity in narrow layers is deleted. Keeping MobileNetV2 as backbone for feature extraction, best performances are achieved for object detection and semantic segmentation.

Tensorflow: TensorFlow is an open source framework developed by Google researchers to run machine learning, deep learning and other statistical and predictive analytics workloads. Like similar platforms, it's designed to streamline the process of developing and executing advanced analytics applications for users such as data scientists, statisticians and predictive modelers.

The TensorFlow software handles data sets that are arrayed as computational nodes in graph form. The edges that connect the nodes in a graph can represent multidimensional vectors or matrices, creating what are known as tensors. Because TensorFlow programs use a data flow architecture that works with generalized intermediate results of the computations, they are especially open to very large-scale parallel processing applications, with neural networks being a common example.

\section{METHODOLOGY}

In order to train a custom face mask detector, we need to break our project into two distinct phases, each with its own respective sub-steps:
Training: Here we'll focus on loading our face mask detection dataset from disk, training a model (using Keras/TensorFlow) on this dataset, and then serializing the face mask detector to disk Deployment: Once the face mask detector is trained, we can then move on to loading the mask detector, performing face detection, and then classifying each face as with_mask or without_mask.

\section{PROPOSED WORKFLOW}

There should be three Python scripts in this Project:

- train_mask_detector.py: Accepts our input dataset and fine-tunes MobileNetV2 upon it to create our mask_detector.model. A training history plot.png containing accuracy/loss curves is also produced

- $\quad$ detect_mask_image.py: Performs face mask detection in static images

- detect_mask_video.py: Using your webcam, this script applies face mask detection to every frame in the stream

Implementing our COVID-19 face mask detector training script with Keras and TensorFlow

Our set of tensorflow.keras imports allow for:

- Data augmentation

- Loading the MobilNetV2 classifier (we will fine-tune this model with pre-trained ImageNet weights)

- $\quad$ Building a new fully-connected (FC) head

- Pre-processing

- Loading image data

We'll use scikit-learn (sklearn) for binarizing class labels, segmenting our dataset, and printing a classification report.

My imutils paths implementation will help us to find and list images in our dataset. And we'll use matplotlib to plot our training curves.

Our command line arguments include:

Dataset: The path to the input dataset of faces and and faces with masks.

Plot: The path to your output training history plot, which will be generated using matplotlib.

Model: The path to the resulting serialized face mask classification model.

1) Training the COVID-19 face mask detector with Keras/TensorFlow 


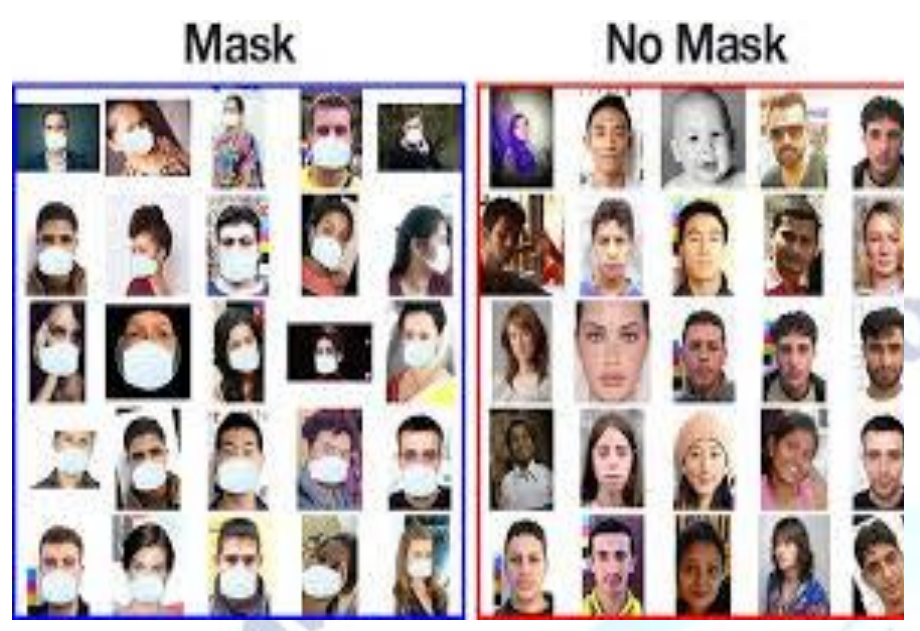

ii. Two-phase COVID-19 face mask detector

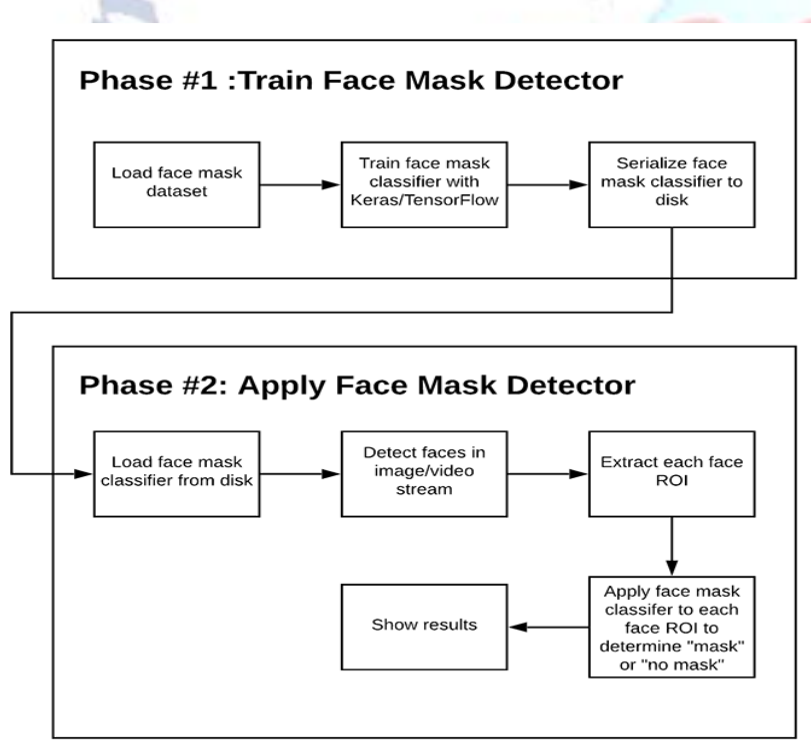

2) We are now ready to train our face mask detector using Keras, TensorFlow, and Deep Learning.

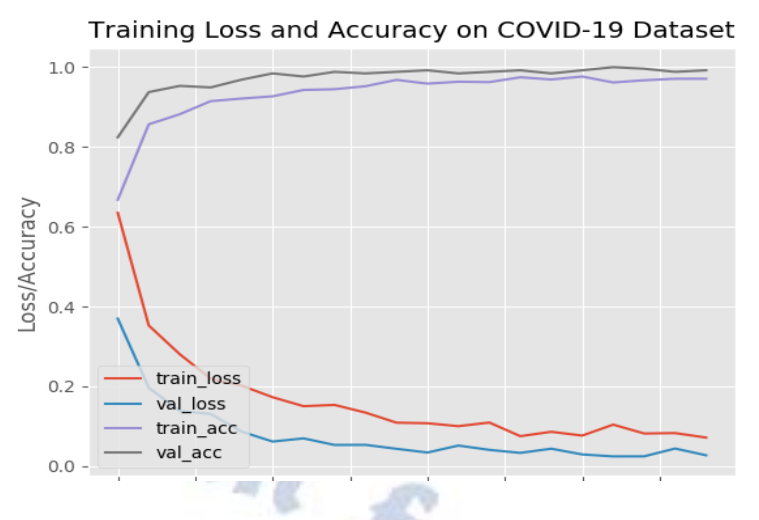

Given these results, we are hopeful that our model will generalize well to images outside our training and testing set.

3) Implementing our COVID-19 face mask detector for images with OpenCV

Now that our face mask detector is trained, let's learn how we can:

- Load an input image from disk

- Detect faces in the image

4) Apply our face mask detector to classify the face as either with_mask or without_mask.

5) Implementing our COVID-19 face mask detector in real-time video streams with OpenCV

This function detects faces and then applies our face mask classifier to each face ROI. Such a function consolidates our code - it could even be moved to a separate Python file if you so choose.

Our detect_and_predict_mask function accepts three parameters:

frame: A frame from our stream

faceNet: The model used to detect where in the image faces are

maskNet: Our COVID-19 face mask classifier model

Inside, we construct a blob, detect faces, and initialize lists, two of which the function is set to return. These lists include our faces(i.e., ROIs), locs(the face locations), and preds(the list of mask/no mask predictions). 


\section{RESULT}

\begin{tabular}{|l|ll|lr|}
\hline Model & Face & & Mask & \\
\hline & Precision & Recall & Precision & Recall \\
\hline Baseline & $89.5 \%$ & $85.2 \%$ & $91.5 \%$ & $88.8 \%$ \\
\hline $\begin{array}{l}\text { COVID Face Mask } \\
\text { +Mobile Net }\end{array}$ & $83.1 \%$ & $95.8 \%$ & $82.5 \%$ & $89.3 \%$ \\
\hline $\begin{array}{l}\text { COVID Face Mask } \\
\text { +Res Net }\end{array}$ & $91.8 \%$ & $96.4 \%$ & $93.2 \%$ & $94.8 \%$ \\
\hline
\end{tabular}

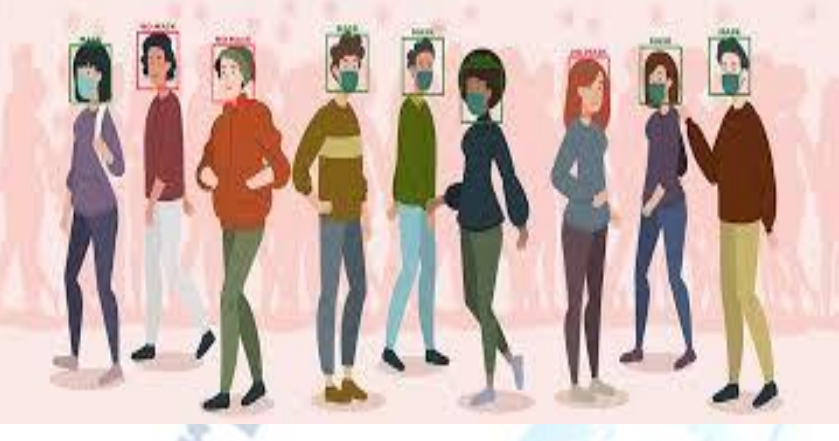

\section{CONCLUSION}

- This Face Mask Detector can be used to analyze whether people are wearing face masks or not in a workplace as well as in a public area and carry out necessary actions to better deal with the pandemic. Automating the task will lead in effective actions taken in short time hence equipping us better to deal with the situation.

- Due to COVID-19 pandemic we need these types of resources to deal with the virus until a vaccine is generated Finally, this Face Mask Detector will make sure that the virus does not spread too much by taking necessary measures like Face Masks.

- Successfully identify the person on image/video stream wearing face mask or not. If the person doesn't wear a mask, the notification will be sent to the respected admin with the help of Python and deep learning algorithm by using the Convolutional Neural Network, Keras Framework and OpenCV.

- This system can therefore be used in real-time applications which require face-mask detection for safety purposes due to the outbreak of Covid-19. This project can be integrated with embedded systems for application in airports, railway stations, offices, schools, and public places to ensure that public safety guidelines are followed.

\section{REFERENCES}

[1] W. H. Organization et al., "Coronavirus disease 2019 (covid-19): situation report, 96,” 2020. 7 A PREPRINT JUNE 9, 2020
[2] P. A. Rota, M. S. Oberste, S. S. Monroe, W. A. Nix, R. Campagnoli, J. P. Icenogle, S. Penaranda, B. Bankamp, K. Maher, M.-h. Chen et al., "Characterization of a novel coronavirus associated with severe acute respiratory syndrome," science, vol. 300, no. 5624, pp. 1394-1399, 2003. [3] Z. A. Memish, A. I. Zumla, R. F. Al-Hakeem, A. A. Al-Rabeeah, and G. M. Stephens, "Family cluster of middle east respiratory syndrome coronavirus infections," New England Journal of Medicine, vol. 368, no. 26, pp. 2487-2494, 2013

[3] Y. Liu, A. A. Gayle, A. Wilder-Smith, and J. Rocklöv, "The reproductive number of covid-19 is higher compared to sars coronavirus," Journal of travel medicine, 2020.

[4] Y. Fang, Y. Nie, and M. Penny, "Transmission dynamics of the covid-19 outbreak and effectiveness of government interventions: A data-driven analysis," Journal of medical virology, vol. 92, no. 6, pp. 645-659, 2020.

[5] N. H. Leung, D. K. Chu, E. Y. Shiu, K.-H. Chan, J. J. McDevitt, B. J. Hau, H.-L. Yen, Y. Li, D. KM, J. Ip et al., "Respiratory virus shedding in exhaled breath and efficacy of face masks."

[6] S. Feng, C. Shen, N. Xia, W. Song, M. Fan, and B. J. Cowling, "Rational use of face masks in the covid-19 pandemic," The Lancet Respiratory Medicine, 2020.

[8] D. Chiang., "Detect faces and determine whether people are wearing mask," https://github.com/AIZOOTech/ FaceMaskDetection, 2020

[9] K. He, X. Zhang, S. Ren, and J. Sun, "Deep residual learning for image recognition," 2016 IEEE Conference on Computer Vision and Pattern Recognition (CVPR), pp. 770-778, 2016.

[10] K. Li, G. Ding, and H. Wang, "L-fen: A lightweight fully convolutional network for biomedical semantic segmentation," in 2018 IEEE International Conference on Bioinformatics and Biomedicine (BIBM), Dec 2018, pp. 2363-2367.

[11] X. Fu and H. Qu, "Research on semantic segmentation of high-resolution remote sensing image based on full convolutional neural network," in 2018 12th International Symposium on Antennas, Propagation and EM Theory (ISAPE), Dec 2018, pp. 1-4.

[12] S. Kumar, A. Negi, J. N. Singh, and H. Verma, "A deep learning for brain tumor mri images semantic segmentation using fcn," in 2018 4th International Conference on Comp.

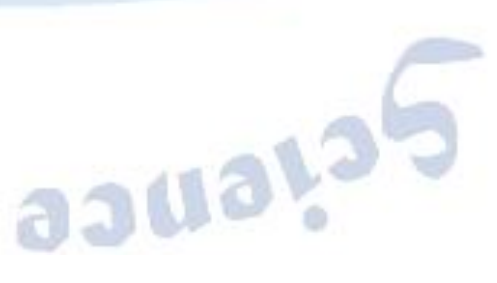

